Under consideration for publication in J. Fluid Mech.

\title{
The effect of wall heating on instability of
}

\author{
channel flow \\ By A. SAMEEN AND RAMA GOVINDARAJAN \\ Engineering Mechanics Unit, Jawaharlal Nehru Centre for Advanced Scientific Research, \\ Bangalore-560064, INDIA
}

(Received ?? and in revised form ??)

\begin{abstract}
A comprehensive study of the effect of wall heating or cooling on the linear, transient and secondary growth of instability in channel flow is conducted. The effect of viscosity stratification, heat diffusivity and of buoyancy are estimated separately, with some unexpected results. ¿From linear stability results, it has been accepted that heat diffusivity does not affect stability. However, we show that realistic Prandtl numbers cause a transient growth of disturbances that is an order of magnitude higher than at zero Prandtl number. Buoyancy, even at fairly low levels, gives rise to high levels of subcritical energy growth. Unusually for transient growth, both of these are spanwise-independent and not in the form of streamwise vortices. At moderate Grashof numbers, exponential growth dominates, with distinct Rayleigh-Benard and Poiseuille modes for Grashof numbers upto $\sim 25000$, which merge thereafter. Wall heating has a converse effect on the secondary instability compared to the primary, destabilising significantly when viscosity decreases towards the wall. It is hoped that the work will motivate experimental and numerical efforts to understand the role of wall heating in the control of channel and pipe flows.
\end{abstract}




\section{Introduction}

One of the well-known methods for delaying a transition to turbulence, for example in boundary layers, has been to reduce the viscosity at the wall. Such a reduction could be brought about by heating or cooling the surface, for example. The objective of this paper is to study the effect of wall heating on the instability of a channel flow. It is shown that heat can have surprising effects on the different mechanisms of transition. We restrict ourselves here to routes based on the linear eigenmodes, a direct nonlinear interaction will be studied in future. The emphasis here is on delaying/advancing the onset of transition to turbulence, rather than drag reduction in full turbulence, as achieved by adding small quantities of polymer.

The critical Reynolds number for linear instability in a plane Poiseuille flow is 5772.22 Orszag (1971)]. However, experiments usually find fully developed turbulence at a much lower Reynolds number, around 1500 [see e.g. Davies \& White (1928); Naravanan \& Naravanan (1967); Patel \& Head (1969); Kao \& Park (1970)]. It is clear that routes to turbulence other than the traditional Tollmien-Schlichting (TS) mechanism are in operation. The background noise in the flow has a major influence in delaying/hastening transition to turbulence, as well as in deciding which mechanism will be dominant Morkovin \& Reshotko (1989)]. At extremely low levels of noise a traditional TS mechanism and/or secondary instability is likely to be followed. At intermediate levels, a transient growth of disturbances is the more likely mechanism for initial disturbance growth Schmid \& Henningson (2001); Meseguer (2002); Foster (1997); Corbett \& Bottaro (2001)]. Once disturbance growth is triggered by a linear mechanism, nonlinearities are required to achieve a new self-sustained state. Alternatively, at higher levels of background noise, nonlinear mechanisms can directly come into play, see e.g. Waleffe (1995); Faisst \& Eckhardt (2003); 
Hof et al. (2004). At present it is not understood exactly which route will be followed when (for a recent review on pipe flow see Kerswell (2005)).

The effect of wall heating on linear stability alone has been studied by several researchers. Here too, the effect of buoyancy has not been clearly quantified. To our knowledge, a detailed study of other mechanisms has not been done. Two related studies of transient growth had different emphasis from the present work. Transient growth in twofluid flow was studied in two-dimensions by Malik \& Hooper (2005) with the objective of understanding the effect of the interface. Biau \& Bottaro (2004) studied transient growth with stable thermal stratification and concluded that such stratification is a viable strategy to control transitional flows. A more detailed retrospective on earlier work is included in the relevant sections later in the text.

We consider two types of heating. The first is asymmetric, with the two walls maintained at different constant temperatures. The second is symmetric, with the walls at one temperature and the fluid at another. Our results may be summarised as follows: the linear stability results are in line with the findings of earlier studies. A decrease in viscosity as one approaches a wall has a large stabilising effect and vice-versa. The effect on the linear eigenmode of reduced heat diffusion (increasing Prandtl number) is extremely small Wall \& Wilson (1996)]. Buoyancy has no effect up to a Grashof number of about 3000 and is enormously stabilising or destabilising thereafter, depending on the sign of the temperature difference. The Rayleigh-Benard and Tollmien-Schlichting modes are distinct at low Grashof number and merge at high Grashof numbers.

The effect of heat on transient growth of instabilities is unexpected. Viscosity stratification, which is the chief player in linear instability, has no discernible effect on this mechanism. Increasing Prandtl number, on the other hand, has an order of magnitude destabilising effect. The assumption that Prandtl number may be neglected in stabil- 
4

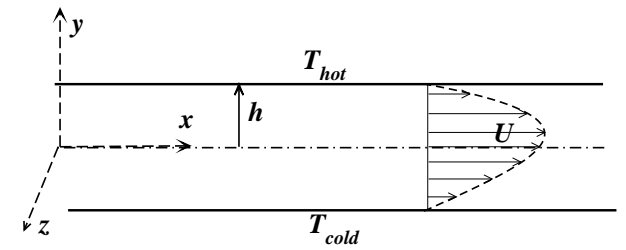

Figure 1. Schematic diagram of the channel, asymmetric heating. The two walls are held at different temperatures, $T_{\text {hot }}$ and $T_{\text {cold }}$, so the mean temperature profile is linear.

\section{A. Sameen and Rama Govindarajan}

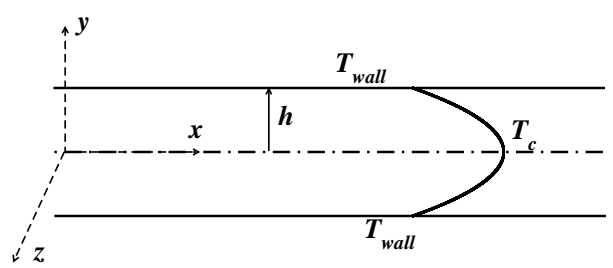

FiguRE 2. Schematic diagram of the channel, symmetric heating. Both walls are maintained at the same temperature, different from that of the fluid.

ity analyses is therefore completely incorrect for this mechanism. Secondary instabilities of the Tollmien-Schlichting modes are usually taken to be unimportant for channel-flow transition, but we find that viscosity-stratification can have a destabilising effect on these modes, which may make them noticeable at large temperature differences.

\section{Basic Velocity Profiles}

Two types of temperature variation, which we shall refer to as the asymmetrically and symmetrically heated cases respectively, are considered, see figures 1 and 2 These provide a fair sample of the type of stratification we may come across. In the first, the two walls of the channel are maintained at different temperatures, $T_{\text {hot }}$ and $T_{\text {cold }}$. At steady state, the temperature within the channel varies linearly between the two. Note that for the unstable Poiseuille-Rayleigh-Benard configuration, the temperature difference $\Delta T$ between the bottom and top walls (and hence the corresponding Richardson and Grashof numbers, defined later) is taken to be positive. The sign of $\Delta T$ is unimportant when buoyancy is neglected.

In the second case, the walls are both maintained at the same temperature, while the incoming fluid is at a different temperature. Such a flow is non-parallel, since the fluid 
temperature downstream tends to equilibrate with the wall temperature, but for large Peclet numbers, the change in the downstream direction is very slow, and the flow may safely be assumed to be locally parallel and the local temperature profile to be parabolic. Stability analyses for more realistic temperature profiles have been conducted, without any qualitative difference. The temperature-dependence of the viscosity is described by the Arrhenius model, which works fairly well for most common liquids like water and alcohol.

$$
\mu(T)=C_{1} \exp \left(C_{2} / T\right)
$$

where $C_{1}$ and $C_{2}$ are constants associated with the fluid under consideration, which is taken in the present computations to be water. The streamwise direction is denoted as $x$, the coordinate $y$ is normal to the wall, and $z$ is the spanwise direction. The mean $x$-momentum equation for a plane parallel channel flow reduces to

$$
\left(\mu U^{\prime}\right)^{\prime}=\frac{d P}{d x} R e
$$

where the primes denote differentiation with respect to $y, R e$ is the Reynolds number defined as $R e=U_{\text {max }} h / \nu_{r e f}, h$ is the half-channel width, $\nu_{r e f}$ is the reference kinematic viscosity, and $d P / d x$ is the constant pressure gradient. The viscosity ratio is defined as $m=\mu_{\text {cold }} / \mu_{\text {hot }}$ for the asymmetric heated case and $m=\mu_{\text {wall }} / \mu_{c}$, where $c$ stands for centerline, for the symmetric case. Knowing $\mu(T)$ and $T(y)$, equation 2.2 is integrated twice by a fourth-order Runge-Kutta method to get $U$. Figure 3 shows typical viscosity and velocity profiles for asymmetric heating. Viscosity profiles for the symmetric case are shown in figure 4 Corresponding velocity profiles and their second derivatives are shown in figure 5

Unless otherwise specified, we define the Reynolds number in terms of the average 
6

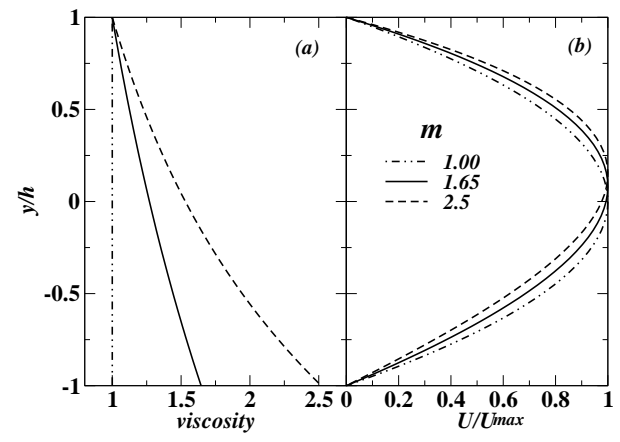

Figure 3. Variation of (a) viscosity and (b) velocity with asymmetric heating. The velocity is scaled by its maximum and the viscosity is scaled here by its value at the hot wall.

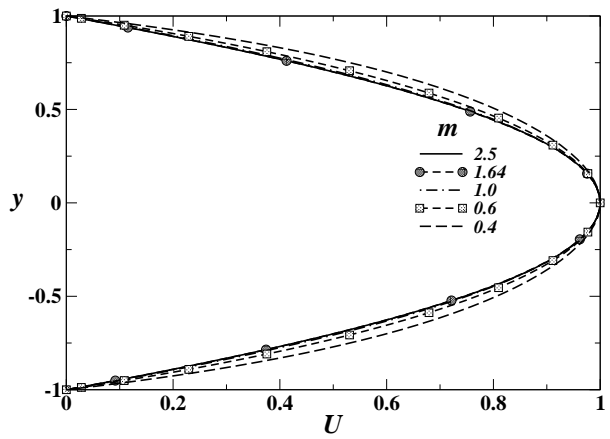

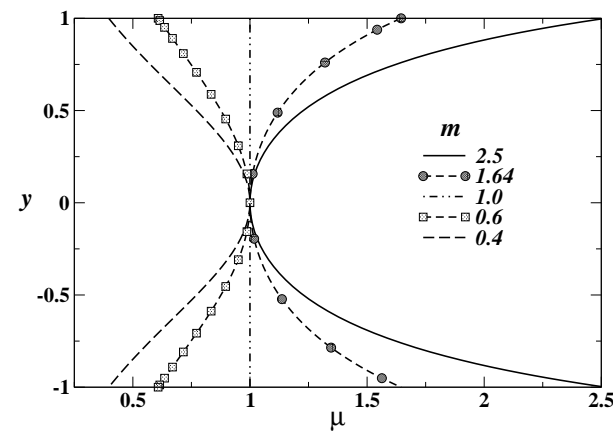

FIGURE 4. Variation of viscosity, scaled here by its value at the centerline, for symmetric heating.

FIGURE 5. Variation of non-dimensional velocity and its second derivative $U^{\prime \prime}$ with symmetric heating.

viscosity across the channel, as follows.

$$
R e \equiv \frac{U_{\max } h}{\int_{-1}^{0} \mu d y}
$$

We can see from Table 1 that the average viscosity varies significantly with increasing $\Delta T$, so defining the Reynolds number as above is approriate for making comparisons at a given Reynolds number between heated and unheated flows. On the other hand, it is seen from the table that the average velocity is practically unchanged by viscosity stratification, so the maximum velocity is a good enough velocity scale. 


\begin{tabular}{cccc}
\hline$\Delta T^{0} K$ & $m$ & $\mu_{\text {avg }}$ & $U_{\text {avg }} / U_{\text {avg }, m=1.0}$ \\
\hline 50 & 0.39 & 0.748 & 1.068 \\
10 & 0.81 & 0.933 & 1.006 \\
0.0 & 1.00 & 1.000 & 1.000 \\
-10 & 1.23 & 1.070 & 0.995 \\
-50 & 2.51 & 1.381 & 0.9944 \\
\hline
\end{tabular}

TABLE 1 . The dependence of the average viscosity $\mu_{a v g}$ on the viscosity ratio, $m$, symmetric heating. The last column shows the ratio of the average mean velocity to its value in the unheated case. The ratio is close to 1.0 in all cases.

\section{Linear stability}

\subsection{The stability equations and their solution}

The disturbance quantities in normal mode form are given as

$$
[\hat{v}, \hat{\eta}, \hat{T}]=[v(y), \eta(y), \hat{T}(y)] \exp [i(\alpha x+\beta z-\omega t)]
$$

where $\hat{v}$ and $\hat{\eta}$ respectively are the components of disturbance velocity and vorticity in the direction normal to the wall, $\hat{T}$ is the disturbance temperature, $\alpha$ and $\beta$ are the wave numbers in the streamwise and spanwise directions respectively, and $\omega$ is the complex frequency of the wave. The linear stability equations may be derived to be

$$
\begin{aligned}
& i \alpha\left[\left(v^{\prime \prime}-\left(\alpha^{2}+\beta^{2}\right)\right)(U-c)-U^{\prime \prime} v\right]=\frac{1}{R e}\left[\mu\left[v^{i v}-2\left(\alpha^{2}+\beta^{2}\right) v^{\prime \prime}+\left(\alpha^{2}+\beta^{2}\right)^{2} v\right]\right. \\
& +\frac{d \mu}{d T} T^{\prime} 2\left[v^{\prime \prime \prime}-\left(\alpha^{2}+\beta^{2}\right) v^{\prime}\right]+\frac{d \mu}{d T} T^{\prime \prime}\left[v^{\prime \prime}+\left(\alpha^{2}+\beta^{2}\right) v\right]+\frac{d^{2} \mu}{d T^{2}} T^{\prime \prime}\left[v^{\prime \prime}+\left(\alpha^{2}+\beta^{2}\right) v\right] \\
& +\frac{d \mu}{d T}\left[U^{\prime} \hat{T}^{\prime \prime}+2 U^{\prime \prime} \hat{T}^{\prime}+\left(\alpha^{2} U^{\prime}+U^{\prime \prime \prime}\right) \hat{T}\right]+2 \frac{d^{2} \mu}{d T^{2}} U^{\prime} T^{\prime} \hat{T}^{\prime}+\frac{d^{2} \mu}{d T^{2}} T^{\prime \prime} U^{\prime} \hat{T} \\
& \left.+\frac{d^{3} \mu}{d T^{3}} U^{\prime} T^{\prime} \hat{T}-\frac{G r}{R e} i \alpha \hat{T}\right] \\
& \alpha(c-U) \eta+i \beta U^{\prime} v=\frac{1}{R e}\left[\mu\left[\eta^{\prime \prime}-\left(\alpha^{2}+\beta^{2}\right) \eta\right]+\frac{d \mu}{d T} T^{\prime} \eta^{\prime}-i \beta \frac{d \mu}{d T}\left(U^{\prime \prime} \hat{T}+U^{\prime} \hat{T}^{\prime}\right)\right.
\end{aligned}
$$




$$
\begin{aligned}
\left.-i \frac{d^{2} \mu}{d T^{2}} T^{\prime} U^{\prime} \hat{T}\right] & \\
& i \alpha\left[(U-c) \hat{T}-T^{\prime} v\right]=\frac{1}{\operatorname{RePr}}\left[\hat{T}^{\prime \prime}-\left(\alpha^{2}+\beta^{2}\right) \hat{T}\right],
\end{aligned}
$$

where $c \equiv \omega / \alpha$. Equations 3.2 and 3.3 respectively are the Orr-Sommerfeld and Squires equations, modified here to account for the effects of viscosity variations, temperature fluctuations and of buoyancy. A Boussinesq approximation has been made. If buoyancy were to be neglected, the above equations would be equivalent to those of Wall \& Wilson (1996).

The Prandtl number is defined as $\operatorname{Pr} \equiv \nu / \kappa$ where $\kappa$ is the coefficient of thermal diffusivity. The Grashof number is $G r \equiv g \gamma \Delta T h^{3} / \nu^{2}, \gamma$ being the volume coefficient of expansion. Equations 3.2 to 3.4 form an eigenvalue problem with the boundary conditions

$$
v( \pm 1)=v^{\prime}( \pm 1)=\eta( \pm 1)=\hat{T}( \pm 1)=0,
$$

and are solved using a Chebyshev collocation spectral method. We perform a temporal stability analysis, the growth rate of the disturbance is obtained from the imaginary part of $c$.

\subsection{Effect of viscosity variation}

In order to isolate the effect of viscosity variation, the Prandtl number and the Grashof number are set to zero. With symmetric heating, we expect that if the viscosity decreases as one approaches the wall, the fuller velocity profile will result in a stabler flow. This expectation is realised, as seen from the neutral stability boundaries in figure [

However, in a channel flow where one wall is maintained at a constant high temperature and the other wall is kept cold, the viscosity decreases towards one wall and increases towards the other. It is not a priori evident what the effect on the linear stability will be. It was found by Potter \& Graber (1972) that any temperature difference between 
the walls is always destabilising. However, Wall \& Wilson (1996) found, using four different viscosity models, that a temperature difference almost always stabilises the flow. The apparent contradiction is because the former work compared results for heated and unheated flow maintaining the input power constant, while the latter made comparisons at a given Reynolds number. Since the flow rate for a given input power is higher for the heated case, the resulting Reynolds number is higher. The stability of viscosity-stratified channel flows was also studied by Pinarbasi \& Liakopoulos (1995); Schafer \& Herwig (1993) with similar conclusions and of boundary layer flows by Craik (1971); Kao (1968); Wazzan et al. (1968, 1979); Strazisar et al. (1977); Schafer et al. (1995).

In the present paper, we define the Reynolds number in terms of average viscosity, and compare results at a given Reynolds number. In agreement with Wall \& Wilson (1996), for asymmetric heating, we find that any temperature difference is stabilising, in terms of the least stable (two-dimensional) linear mode (figure 7). We have confirmed Sameen (2004)] that the production of disturbance kinetic energy is reduced at the cold wall and increased at the hot wall compared to the unheated case. The dissipation is similar in all cases. The highly oblique modes, unlike the two-dimensional ones, are practically unaffected (not shown). This observation will assume significance when we discuss transient growth.

\subsection{Effect of heat diffusivity}

We know that for liquids such as water, heat diffuses slower than momentum, so the assumption of $\operatorname{Pr}=0$ is not justifiable. Surprisingly however, the linear stability, as measured by the least stable eigenmode, is practically unaffected by a decrease in heat diffusivity Wall \& Wilson (1996)]. Present computations confirm this (figure 8). However, the prevalent conclusion that heat diffusivity does not affect flow stability, and therefore that the Peclet number may be set to zero in stability analyses, is shown in the 


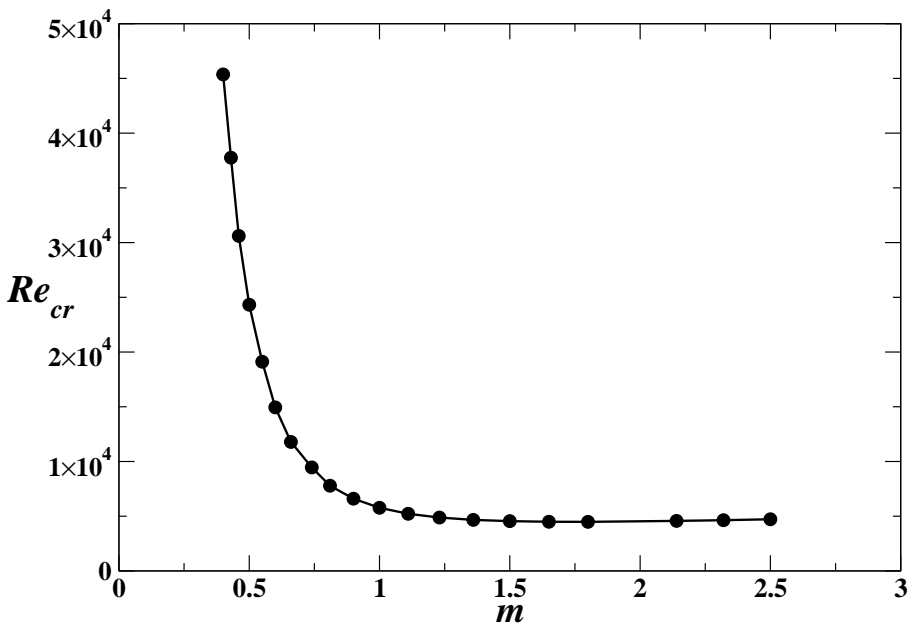

FiguRE 6. Effect of viscosity variation on linear stability, symmetric heating.

For unstratified flow, i.e., at $m=1.0, R e_{c r}$ is 5772.2 .

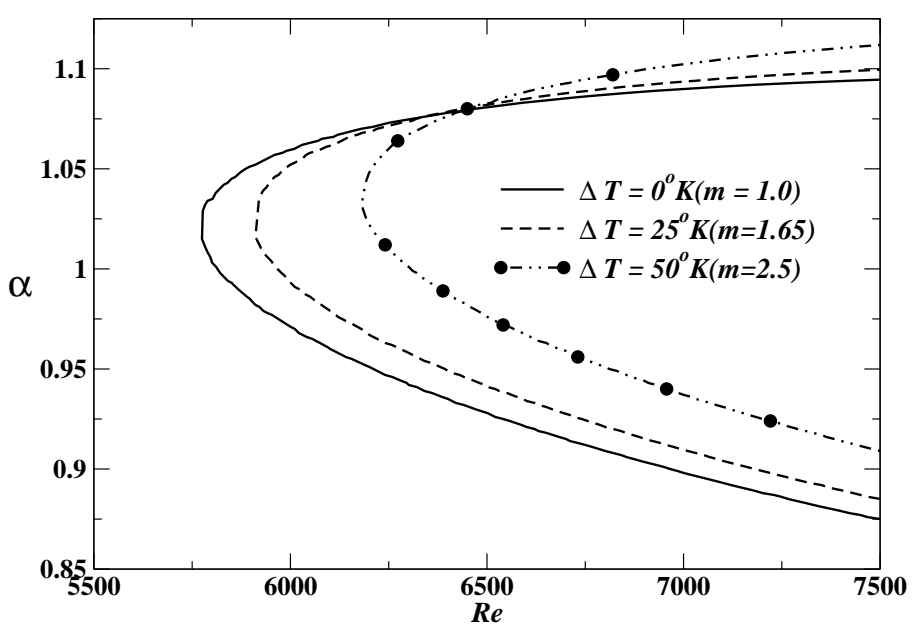

FIGURE 7. Stability boundaries for various viscosity ratios. Asymmetric heating,

$$
T_{\text {cold }}=295^{\circ} \mathrm{K}
$$

next section to be incorrect. Increasing the Prandtl number to $O(1)$ vaules can enhance transient growth by an order of magnitude.

\subsection{Effect of buoyancy: the Poiseuille-Rayleigh-Benard problem}

We consider the asymmetrically heated case here. When the upper wall is cold relative to the lower one, the resulting unstable stratification of density leads, at low flow rates, 


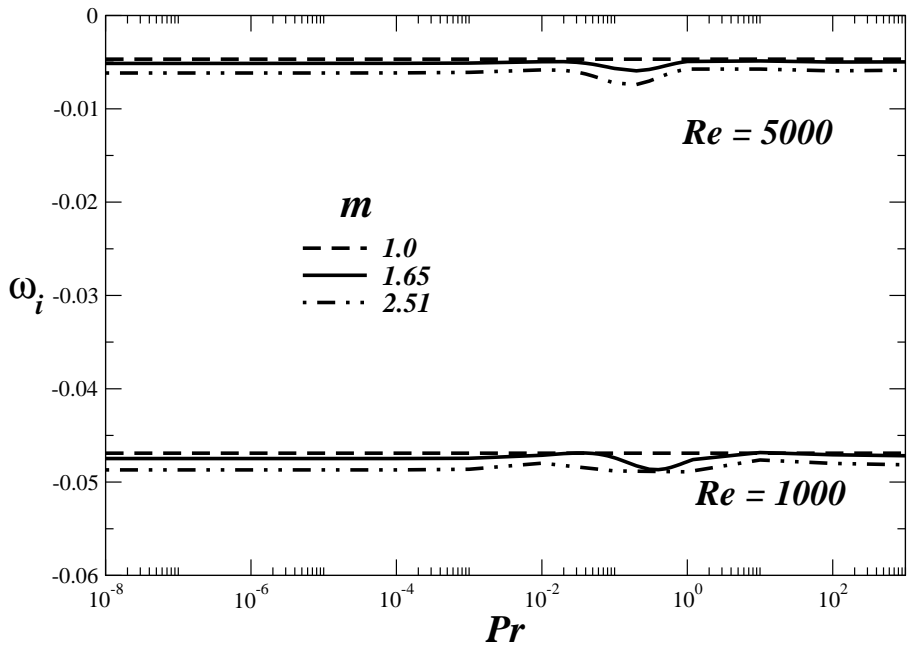

Figure 8. Most unstable eigenvalue at various Prandtl numbers for different $\Delta T$ at $\alpha=0.9$ and $R e=1000,5000$. The effect of Prandtl number is negligible.

to a buoyancy driven instability similar to the Rayleigh-Benard Chandrasekhar (1961); Turner (1959); Platten \& Legros (1984)]. The effect of mean shear on this instability has been studied by Zhang et al. (1998); Deardorff (1965), for example, and this problem been studied by Zhang et al. (1998); Deardorff (1965), for example, and this problem is reviewed in Platten \& Legros (1984); Mahajan et al. (1988). On the other hand, the effect of buoyancy on the Tollmien-Schlichting modes in plane Poiseuille flow has been investigated by Gage \& Reid (1968); Gage (1971); Tveitereid (1974) and Fuiimura \& Kellv (1988). Several approximations were made in these early studies. For example, viscosity variations were neglected and the base flow was taken to be parabolic. It was found that a critical Reynolds number always exists for any level of density stabilisation, while there exists a Richardson number $\left(R i \equiv G r / R e^{2}\right)$ above which the flow is stable for all Reynolds number.

In figure 9 the critical Reynolds number, $R e_{c r}$ for a temperature difference of $25^{\circ} \mathrm{K}$ is plotted for various Richardson numbers. The trends are the same as in Gage \& Reid (1968) and Tveitereid (1974), but there are minor numerical discrepancies, which we attribute to the more appropriate velocity and viscosity profiles used here. The effect of 


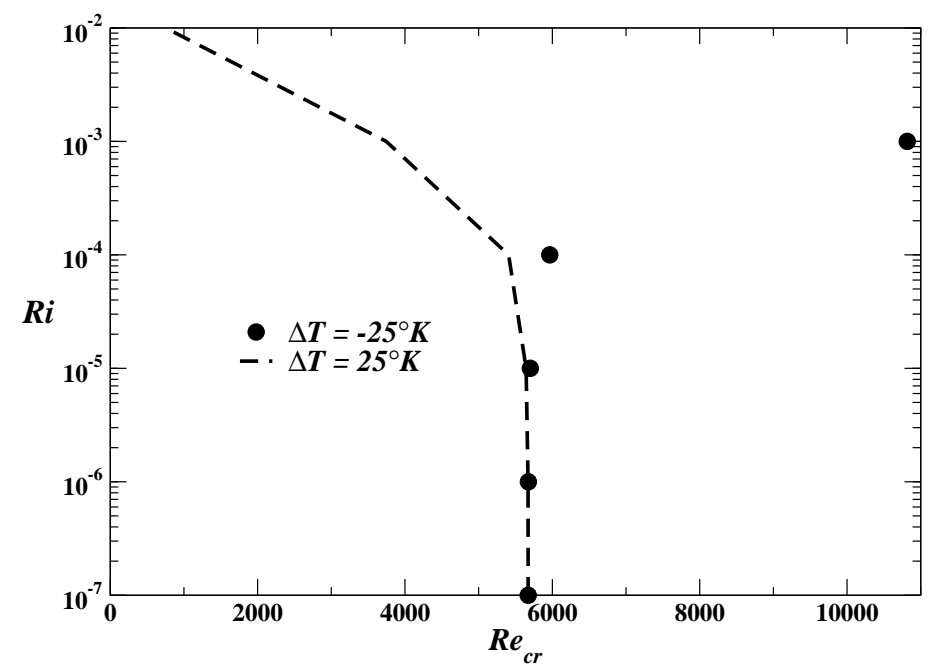

FiguRE 9. Neutral stability Reynolds number as a function of Richardson number, $\operatorname{Pr}=1.0$. Note that for stable stratification $(\Delta T<0)$, the Richardson number is negative, its absolute value is plotted here. The portion to the right of the neutral points is unstable.

buoyancy are negligible when the Richardson number is below $10^{-4}$, and of either sign. At higher Richardson numbers, for unstable stratification, figure 9 shows that the flow is highly destabilised. The stability boundaries are plotted in figure 10 in terms of the Grashof number, a given Grashof number being more simple to achieve experimentally. Distinct modes of Rayleigh-Benard type and of Tollmien-Schlichting type are evident at intermediate levels of $G r$. The modes merge at Grashof numbers above $\sim 25000$. The numerical value at the bifurcation point varies slightly with Prandtl number and temperature difference. The unstable region in the Grashof-Reynolds parameter space is shown in figure 11

We have discussed the stability in terms of the most unstable linear mode. However, a transient growth of decaying modes can often be the dominant mechanism of transition to turbulence in channel flows. We shall see in the next section that the effect of heat on flow instability throws up several surprises. 


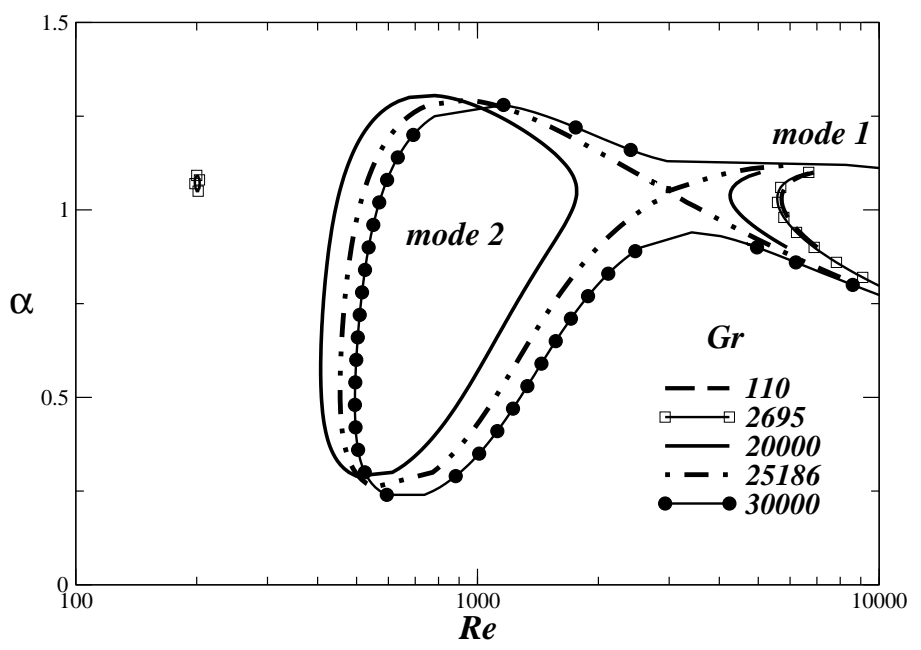

FIgURE 10. The neutral curves for unstably thermal stratified flow at Prandtl number 7.0, $\Delta T=25^{\circ} \mathrm{K}$. The second (Rayleigh-Benard-like) mode starts appearing at $G r=2695$ and merges with the Tollmien-Schlichting mode at $G r=25186$.

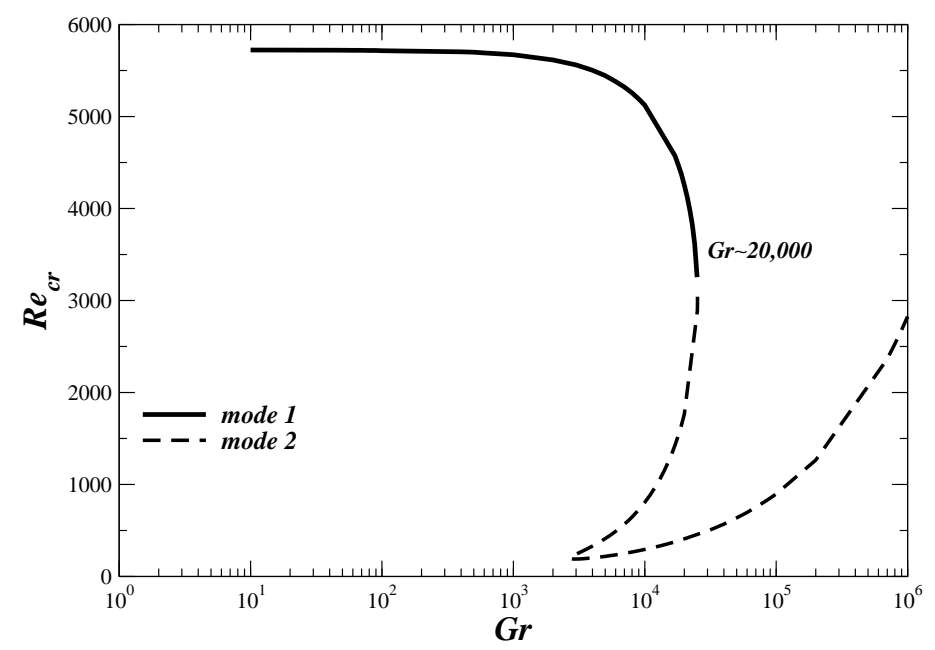

FiguRE 11. A consolidated picture of the variation of the critical Reynolds number with the Grashof number. The Tollmien-Schlichting mode is shown by the solid line, and the Rayleigh-Benard mode by the dashed line. The region above the curves is unstable.

\section{Transient Growth}

The linear stability operator is not self-adjoint, and the resulting non-orthogonality of the eigenfunctions is known to be able to give rise to large levels of transient growth of dis- 
turbance kinetic energy even when all individual eigenmodes are stable. In wall-bounded flows, transient growth is mainly caused by the interaction between the Orr-Sommerfeld and Squire modes Reddv \& Henningson (1993); Criminale et al. (2003)] from the coupling term, $-i \beta U^{\prime}$, appearing in Squire's equation. The most likely structures arising due to transient growth are streamwise streaks Reddv et al. (1998); Reddv \& Henningson (1994, 1993); Schmid \& Henningson (2001)]. We use the standard approach for computing the maximum transient growth.

The effect of viscosity-stratification, in contexts other than heat Malik \& Hooper (2005); Chikkadi et al. (2005)] has been addressed before, though not completely. The effect of buoyancy has been studied under stable stratification alone by Biau \& Bottaro (2004). The effect of heat diffusivity on transient growth has not been studied before, to our knowledge.

The disturbance kinetic energy, $g(t)$ Schmid \& Henningson (2001)], is written as

$$
g(t)=\frac{\|\kappa(t)\|_{E}^{2}}{\|\kappa(0)\|_{E}^{2}}=\frac{\left\|e^{-i \Lambda t} \kappa(0)\right\|_{E}^{2}}{\|\kappa(0)\|_{E}^{2}}
$$

Its time evolution is represented by the matrix $\partial \kappa / \partial t=-i \Lambda \kappa$, where $\kappa=\left(\kappa_{1}, \kappa_{2}, \ldots, \kappa_{N}\right)^{T}$ and $\Lambda=\operatorname{diag}\left\{\omega_{1}, \omega_{2}, \ldots \omega_{N}\right\}$, where $\kappa_{j}$ is the $j^{t h}$ expansion coefficient of the eigenfunctions of the linear modes which are the dominant contributors here. The superscript $T$ denotes transpose. Maximising equation (4.1) for all possible initial conditions $\kappa(0)$,

$$
G(t)=\max _{\kappa \neq 0} g(t)
$$

We then define $G_{\max }$ as the maximum over time of $G(t)$ for one particular $R e, \alpha$ and $\Delta T$, see figure 12

The contour plot for $G_{\max }$ for unheated flow is shown at $R e=1000$ in figure 13 for comparison with the results for heated flow to follow. A maximum growth of $G_{\max }=196$ is obtained for $\alpha=0.0$ and $\beta=2.05$ [see Reddv \& Henningson (1993)] ]. 


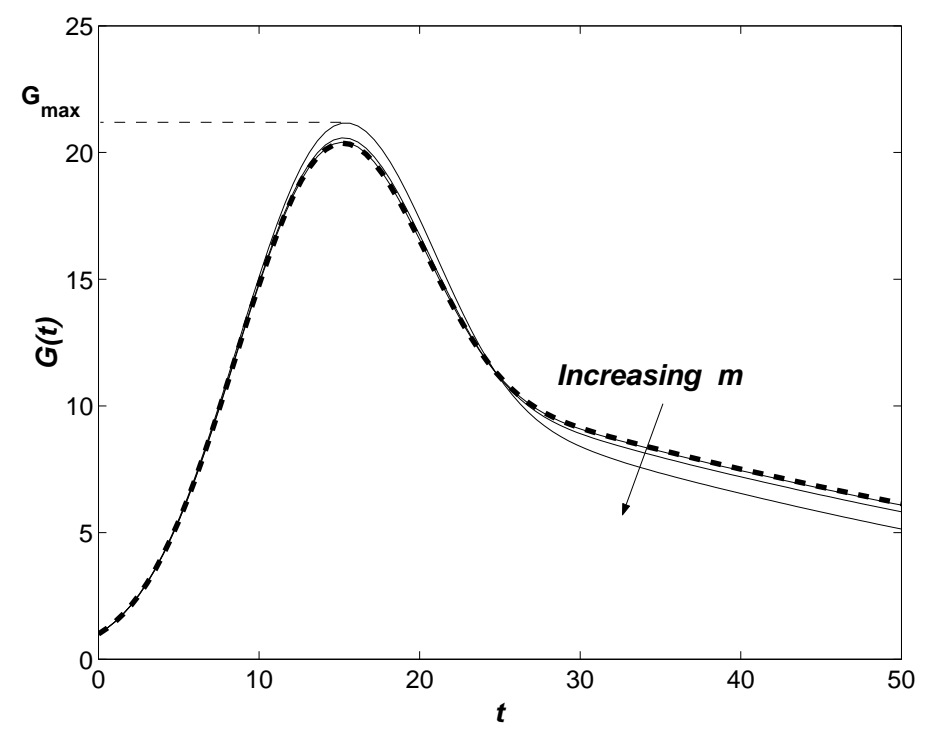

FiguRE 12. The energy amplification evolution for various viscosity ratios for $R e=3000, \alpha=1$, asymmetric heating. The thick dashed curve is for unstratified flow, and the solid lines are for $m=1.2,1.65$ and 2.5 in the order indicated in the figure.

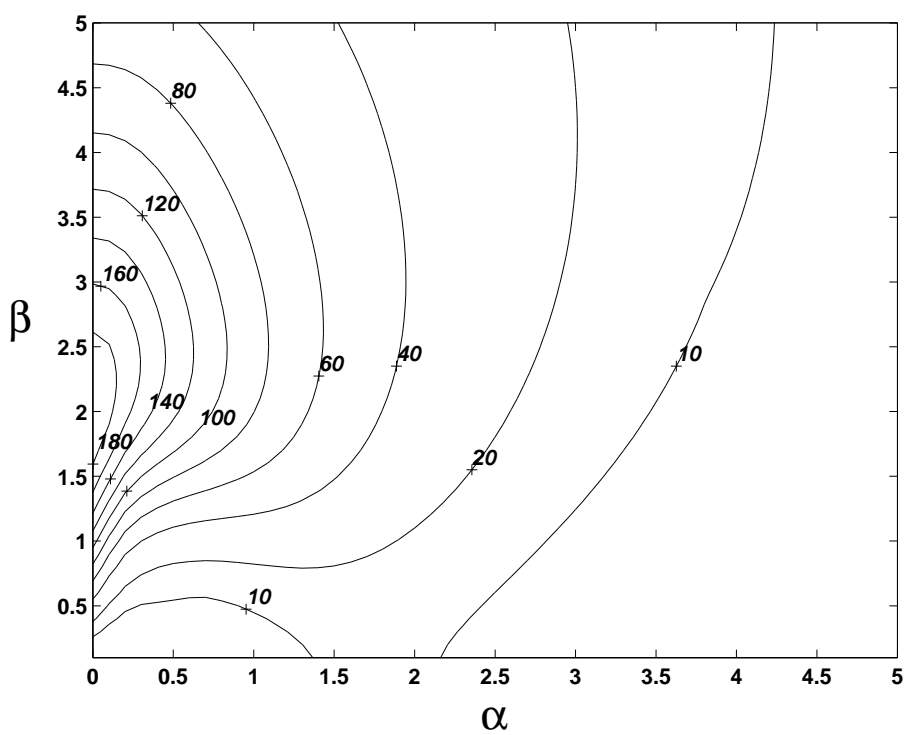

FiguRE 13. The contour of $G_{\max }$ (the maximum over time of $G(t)$ ) for $R e=1000$ in the $\alpha-\beta$ plane, $\Delta T=0^{\circ} K$. This matches well with Reddv \& Henningson (1993) 


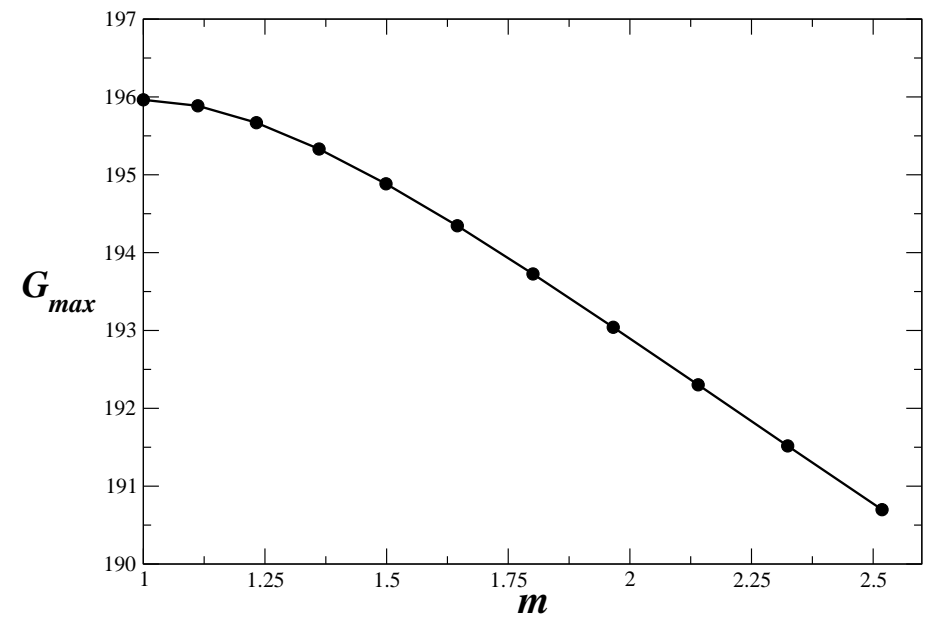

FIgURE 14. The variation of $G_{\max }$ at $\alpha=0$ and $\beta=2$ for various viscosity ratios at $R e=1000$, asymmetric heating. The maximum deviation of $G_{\max }$ from the unheated value of 196 is only $3 \%$.

\subsection{Effect of viscosity stratification}

As before, we first take the Prandtl number to be zero, i.e., assume that temperature fluctuations diffuse away instantaneously. We also neglect buoyancy, in order to isolate the effect of viscosity stratification alone. For the asymmetrically heated case, the growth of kinetic energy is seen in figure 12 to change only marginally with heating. The example shown in figure 12 is for $R e=3000$ and $\alpha=1$, but viscosity stratification has very little effect on transient growth at any value of $R e$ and $\alpha$. The effect of asymmetric heating is quantified in figure 14 in terms of $G_{\max }$ at $\alpha=0$ and $\beta=2$. There is a marginal stabilisation with viscosity stratification. This result is in line with the result for linear stability, but much smaller in magnitude. The contours of $G_{\max }$ for symmetric heating are plotted in figures [15] in the $\alpha-\beta$ plane, and the variation of $G_{\max }$ with viscosity ratio is plotted in figure 16 at $\alpha=0$. There is again a slight stabilisation with increase in viscosity stratification.

The insignificant effect of viscosity stratification is consistent with our recent study 


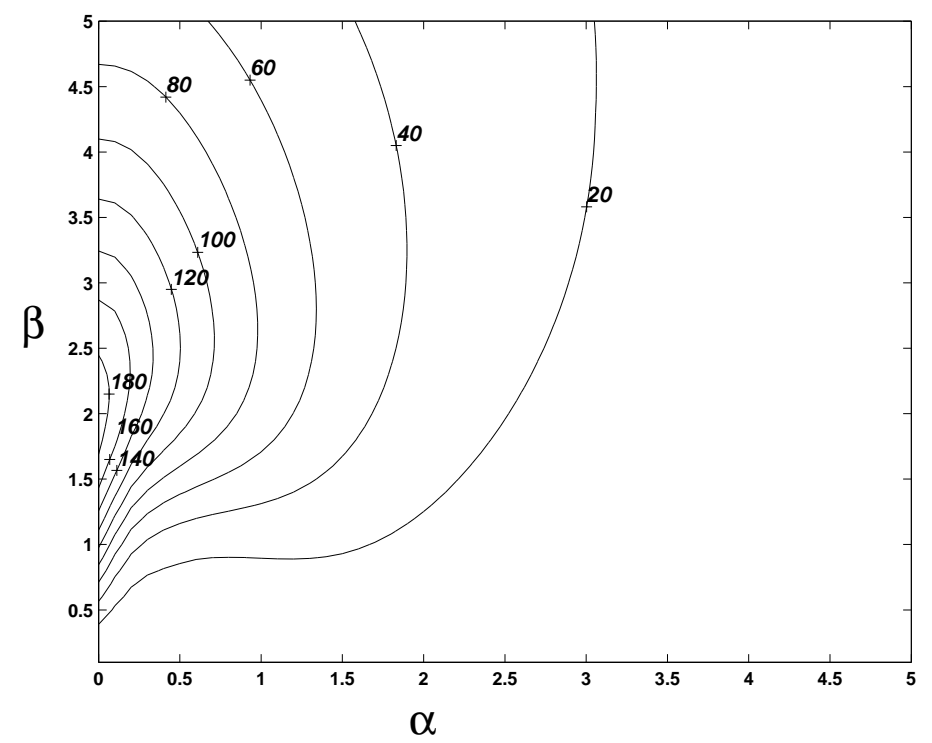

Figure 15 . The contour of $G_{\max }$ for symmetric heating at $R e=1000$ again in the $\alpha-\beta$ plane, $m=0.4$.

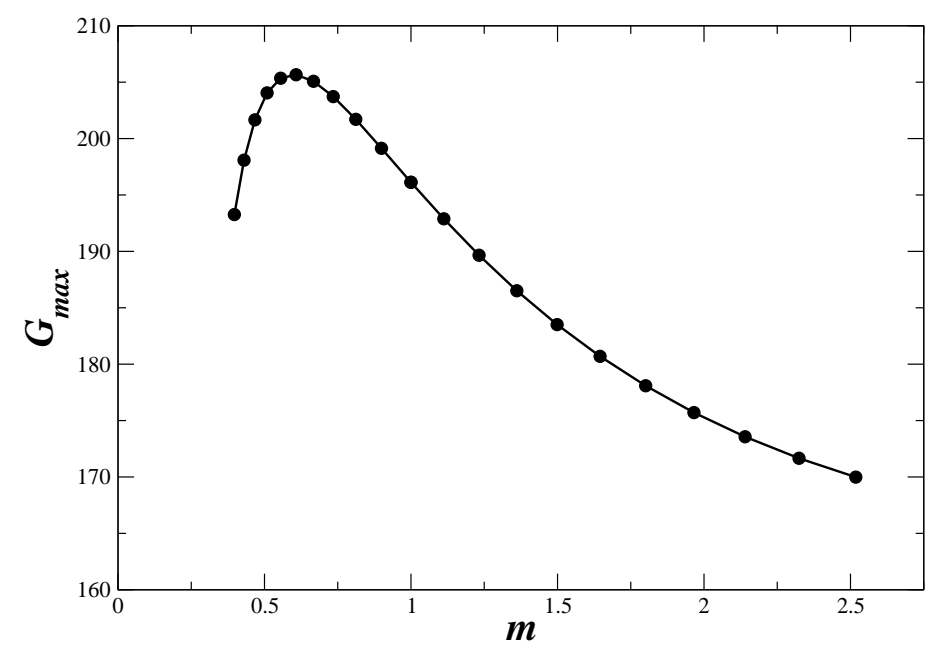

Figure 16. The $G_{\max }$ variation at $\alpha=0.0$ and $\beta=2.0$ for various viscosity ratios at $R e=1000$, symmetric heating. The maximum deviation of $G_{\max }$ from that for unstratified flow is only $13 \%$.

of transient growth in two-fluid and non-Newtonian flows Chikkadi et al. (2005)]. As discussed there, the $U^{\prime \prime}$ term, which affects the least stable eigenmode dramatically, has no effect on streamwise vortices arising from $\alpha=0$, which dictate transient growth. The 

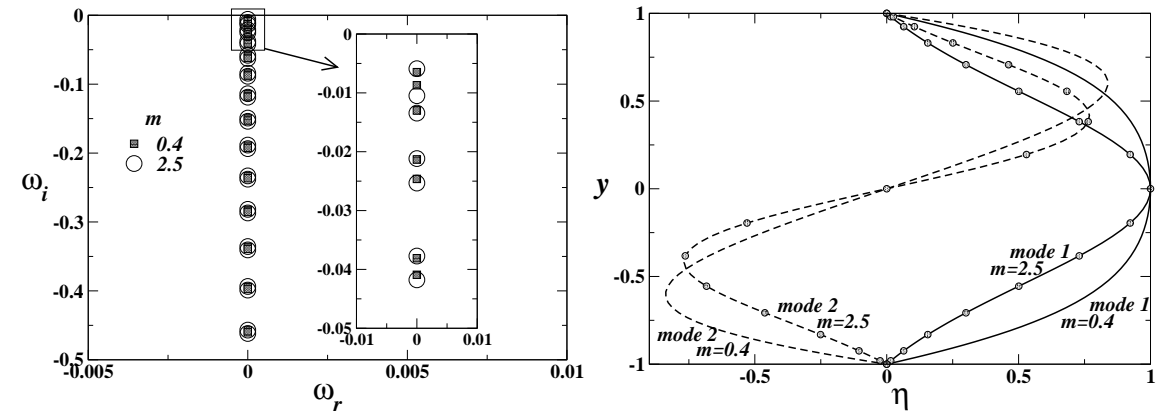

Figure 17. (a) The eigenspectra for two extremes of viscosity stratification, $m=0.4$ and 2.5 , for $\alpha=0, R e=1000$ and $\beta=2.0$, symmetric heating. (b) The corresponding eigenfunctions of the first two unstable eigenvalues in each case.

eigenspectrum, and typical eigenfunctions at $\alpha=0$ are shown in figure 17 to be very similar at two extremes of viscosity stratification. Equation 3.3 drives the dynamics rather than equation 3.2 under these conditions, and the terms containing viscosity gradients have been verified numerically to be small.

\subsection{Effect of heat diffusivity}

It has been seen that the Prandtl number has a marginal effect on the most unstable linear mode. In contrast, we find here that reducing heat diffusivity has a large destabilising effect on the transient growth of disturbance kinetic energy. A dimensionless quantity for measuring growth is the energy norm defined as

$$
E=\int\left|u_{p}\right|^{2}+\left|v_{p}\right|^{2}+\left|w_{p}\right|^{2}+|\hat{T}|^{2} d y
$$

There is some flexibility in defining the measure, but the results are not expected to change qualitatively Hanifi \& Henningson (1998); Biau \& Bottaro (2004)]. In figure 18 for a temperature difference of $25^{\circ} \mathrm{K}$ at a Reynolds number of 1000 the effect of Prandtl number is shown. As the Prandtl number is increased from $10^{-4}$ to 1 , the transient growth is seen to increase dramatically. The large destabilisation comes from a new two- 

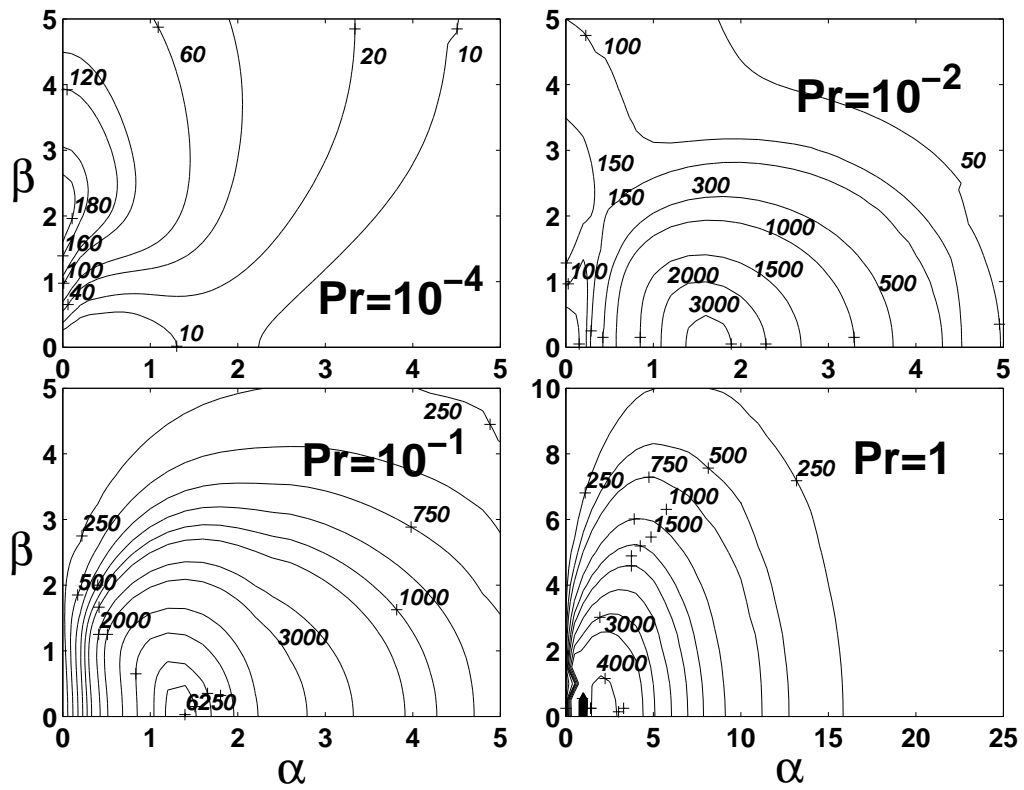

Figure 18. Asymmetric heating: contour plot of $G_{\max }$ for $T=25^{\circ} \mathrm{K}, \mathrm{Re}=1000$ for various Prandtl numbers. Note that for $\operatorname{Pr}=1$ the scale employed is different.

dimensional transient growth. This is true for symmetric heating as well (not shown). We now have a situation where transient growth dominates, but not via the standard streamwise streaks and streamwise vortices.

\subsection{Effect of unstable density stratification}

In their studies of stable thermal stratification Biau \& Bottaro (2004) have found that as stratification increases flow becomes increasingly stable, both in terms of exponential growth as well as transient growth. Viscosity variations were not accounted for in their calculation. In this paper, we concentrate on unstable thermal stratification.

Figures 19 to 22 show contour plots of maximum growth of transient energy for various Grashof numbers at increasing Prandtl number for a temperature difference of $\Delta T=$ $25^{\circ} \mathrm{K}$. As expected, when heat diffusivity is high, buoyancy has little effect. For $\mathrm{Pr} \leqslant$ 0.01, the Prandtl number dictates the instability, and buoyancy has very little effect. At $\operatorname{Pr}=1$, however, the situation is completely different. For Grashof numbers of 1000 and 

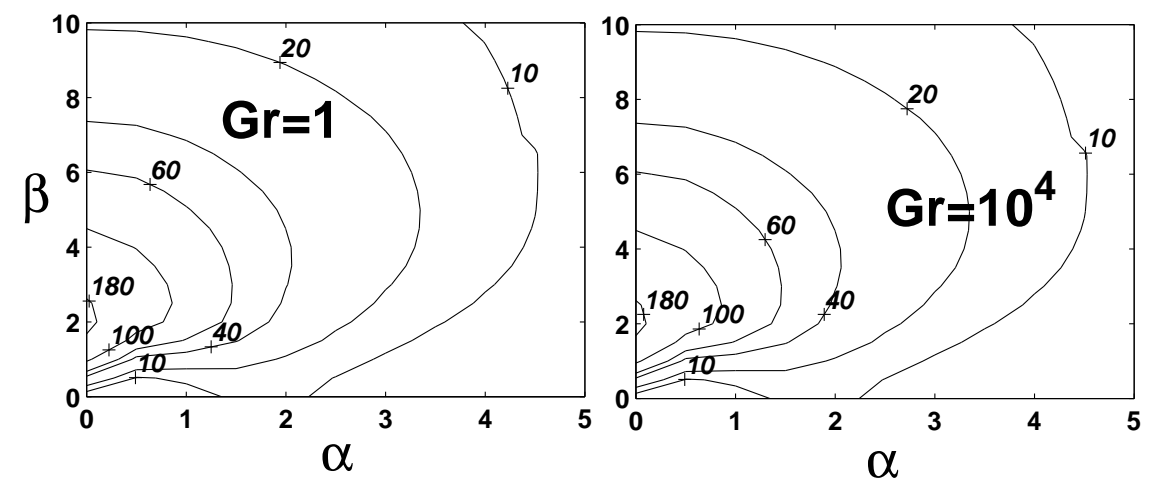

Figure 19. The transient growth at various Grashof number for $\Delta T=25^{\circ} \mathrm{K}$, $R e=1000$ and $\operatorname{Pr}=10^{-4}$. Since temperature perturbations diffuse away rapidly, buoyancy does not have much effect.
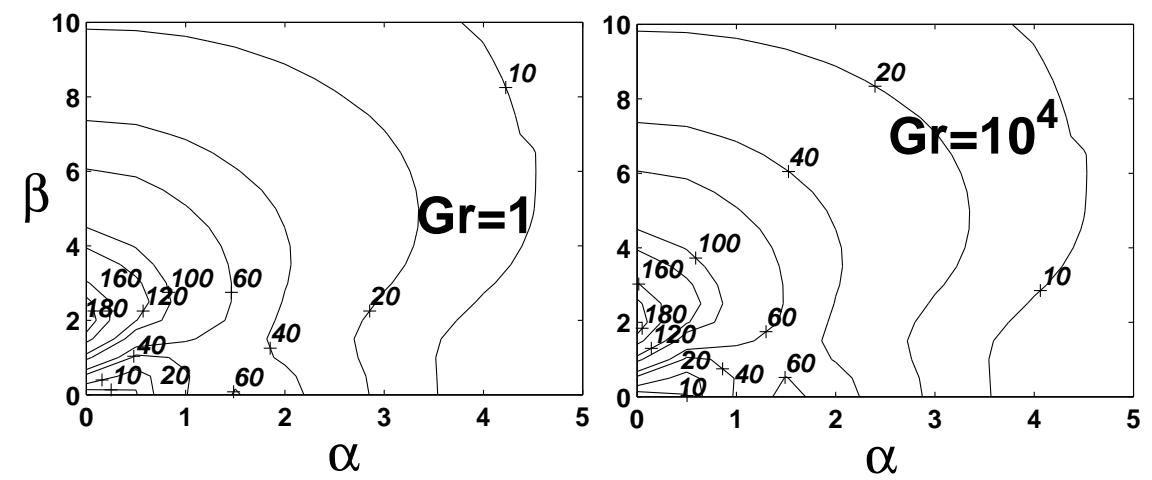

Figure 20. Same as figure 19 but for $\operatorname{Pr}=10^{-3}$. The Grashof number has no effect upto a value of $\sim 10^{4}$. At $G r=10^{4}$ a new growth appears at $\beta=0$, which will dominate at higher Prandtl number.

below we see extremely large levels of subcritical transient growth. This growth is twodimensional. Above this Grashof number of course, a linearly unstable mode exists. The transient growth in unheated channel flow is well known to display itself as streamwiseindependent structures, like streaks and vortices. Our results indicate that such structures will not be much in evidence in heated flows at realistic Prandtl or Grashof numbers. Rather, a spanwise-independent growth occurs. Experimental and numerical verification 

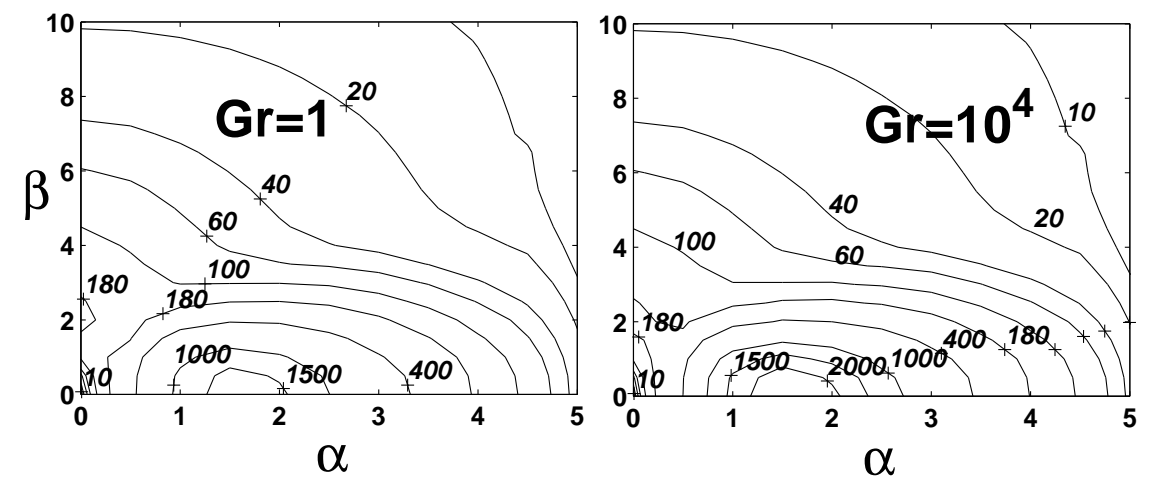

Figure 21. Same as figure 19 but for $\operatorname{Pr}=10^{-2}$. The new subcritical mode is now dominant at low Grashof number as well. The spanwise-independence of the largest transient growth, making the Poiseuille-Rayleigh-Benard problem essentially two-dimensional, is unusual.

of this kind of transient growth could have interesting consequences for wall heating as a control option.

\section{Secondary Instability}

A flow containing linear modes (either growing or decaying) of sufficient amplitude $A_{p}$ can become unstable to new secondary modes of instability. In unheated channel flow, secondary instability is considered unimportant, since it may only play a role when external disturbance levels are extremely small. We show here that viscosity variations can significantly destabilise the secondary mode, thus making it more relevant to the transition process. The Prandtl number and Grashof number are set equal to zero.

The approach here is as in Herbert (1983) and Bavly et al. (1988). All flow variables are decomposed in the form

$$
u(x, y, z, t)=U(y)+A_{p} \hat{u}_{p}(x, y, t)+A_{s} \hat{u}_{s}(x, y, z, t),
$$

where $\hat{u}_{p}$ is the linear instability mode of the previous section. A subscript $p$ has been introduced in this section to denote primary mode of instability. $A_{s}$ is the amplitude of 

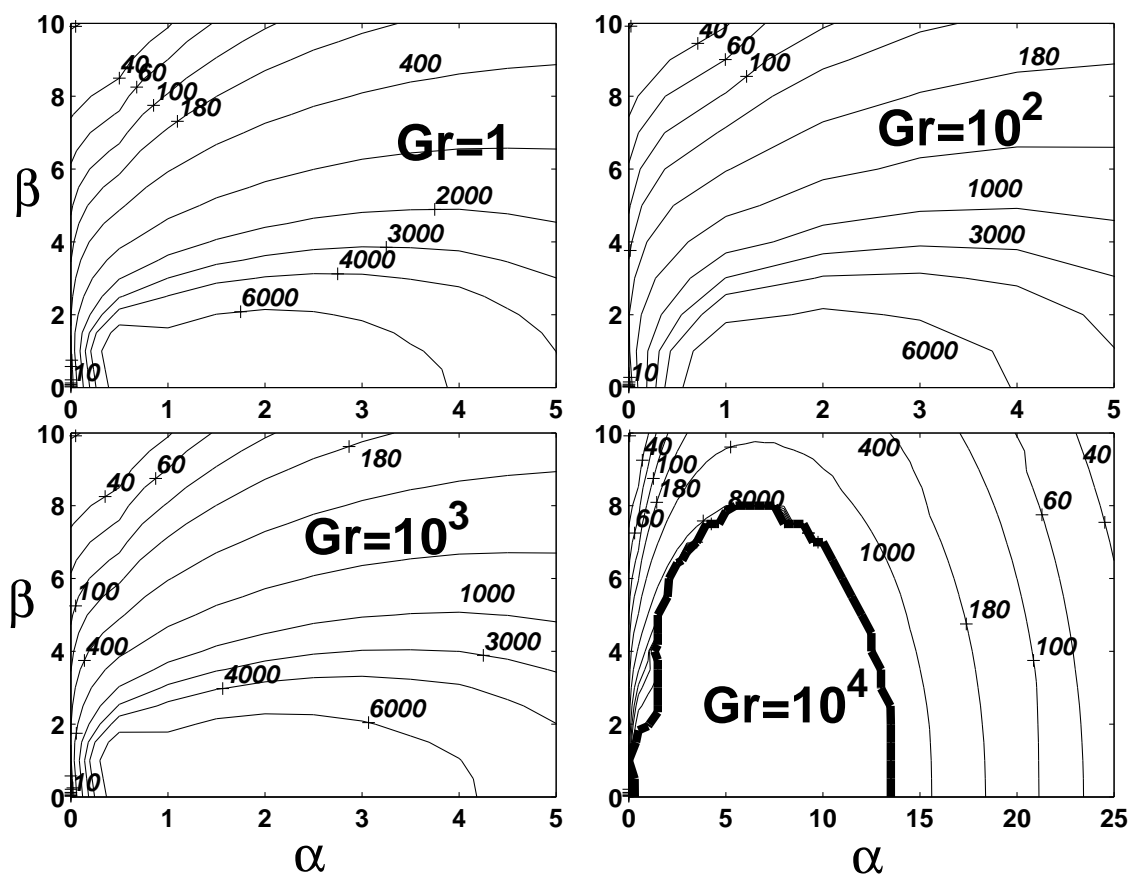

Figure 22. Same as figure 19 except that $\operatorname{Pr}=1$. The transient growth is even higher than before. The region inside the thick curve for $G r=10^{4}$ is linearly unstable.

the secondary. Note that since only the least stable linear modes are relevant here, it is sufficient to consider two-dimensional primary modes, by Squire's theorem.

The secondary perturbation quantities are assumed to be of the form

$$
\begin{aligned}
& \left(\hat{u}_{s}, \hat{v}_{s}, \hat{w}_{s}\right)=\frac{1}{2}\left[\left(u_{+}, v_{+}, w_{+}\right)(y, t) e^{i\left(\alpha_{+} x+\beta_{s} z\right)}\right. \\
& \left.+\left(u_{-}, v_{-}, w_{-}\right)(y, t) e^{i\left(\alpha_{-} x-\beta_{s} z\right)}+\text { c.c. }\right]
\end{aligned}
$$

where $\alpha_{+}$and $\alpha_{-}$are the wave numbers of the secondary waves in the streamwise direction, $\beta_{s}$ is the wave number in the spanwise direction. The direct interaction between primary waves is assumed to be negligible. For the flow under consideration, the growth/ decay rates are so small that $d A_{p} / d t$ can be neglected during one period of time, and the primary flow may be taken to be periodic. Substituting these decompositions in the momentum equations, eliminating pressure and neglecting non-linear terms in the secondary 
disturbance, we arrive at the secondary disturbance equations. On averaging these over $x, z$ and $t$, only the resonant modes survive, which are given by

$$
\alpha_{+}+\alpha_{-}=\alpha
$$

The cases of $\alpha_{+}=\alpha / 2$ and $\alpha_{+}=\alpha$ are called the subharmonic and the fundamental modes respectively. Using continuity the streamwise component of secondary disturbance velocity is eliminated and we get the secondary perturbation equations:

$$
\begin{aligned}
& -D \frac{\partial v_{+}}{\partial t}+s \frac{\partial f_{+}}{\partial t}=-s A f_{+}+\left(A D-i \alpha_{+}(D U)\right) v_{+}-A_{p}\left[\frac{i \alpha_{+}^{2}}{2 \alpha_{-}} u_{p} D+\frac{v_{p} \alpha_{+} D^{2}}{2 \alpha_{-}}\right. \\
& \left.+\frac{i\left(D u_{p}\right) \alpha_{+}}{2}\right] v_{-}^{*}+\frac{A_{p} \alpha_{+}^{2}}{2}\left[-v_{p} D+i \alpha_{-} u_{p}+\frac{i \beta_{s}^{2}}{\alpha_{-}} u_{p}+\frac{\beta_{s}^{2}}{\alpha_{+} \alpha_{-}} v_{p} D\right] f_{-}^{*} \\
& \quad \frac{\partial v_{+}}{\partial t}-D \frac{\partial f_{+}}{\partial t}=-A v_{+}+(A D+(D A)) f_{+}-\frac{A_{p}\left(\alpha+\alpha_{-}\right)}{2}\left[\frac{v_{p}}{\alpha_{-}} D-i u_{p}\right] v_{-}^{*} \\
& +\frac{A_{p}}{2}\left[-i\left(\alpha+\alpha_{-}\right) u_{p} D-i \alpha_{-}\left(D u_{p}\right)+v_{p}\left(\frac{\alpha_{+} \beta_{s}^{2}}{\alpha_{-}}+D^{2}\right)\right] f_{-}^{*}
\end{aligned}
$$

where $A=\left[i \alpha_{+} U+\mu s-\mu d^{2}-\mu^{\prime} D\right], f_{+}=-\frac{i}{\beta_{s}} w_{+}, D=d / d y$. Equations (5.4) \& (5.5) and complementary equations for $v_{-}^{*}$ and $f_{-}^{*}$ are solved using a Chebyshev collocation spectral method, with the boundary conditions $\hat{u}_{s}, \hat{v}_{s}, \hat{w}_{s}=0$ at $y= \pm 1$. The dispersion relation is $F\left(A_{p}, \beta_{s}, m, R e, \alpha, c,\right)=0[$ see Herbert (1983)]. The growth rate is highly sensitive to the primary amplitude level $A_{p}$, and increases with increasing $A_{p}$. The present computations are validated by comparing with the unstratified case in Herbert (1983) as discussed in Sameen (2004).

\subsection{Asymmetric heating}

A value of $A_{p}=0.01$ is taken, to be representative of an intermediate level of primary disturbance. The variation of secondary growth rate $\omega_{i s}$ with the spanwise wave number for various viscosity ratios is plotted in figures 23 As the temperature difference increases, a second highly unstable mode appears. This mode is three-dimensional, the spanwise and streamwise wavelengths being very close to each other. The modes which are closer 


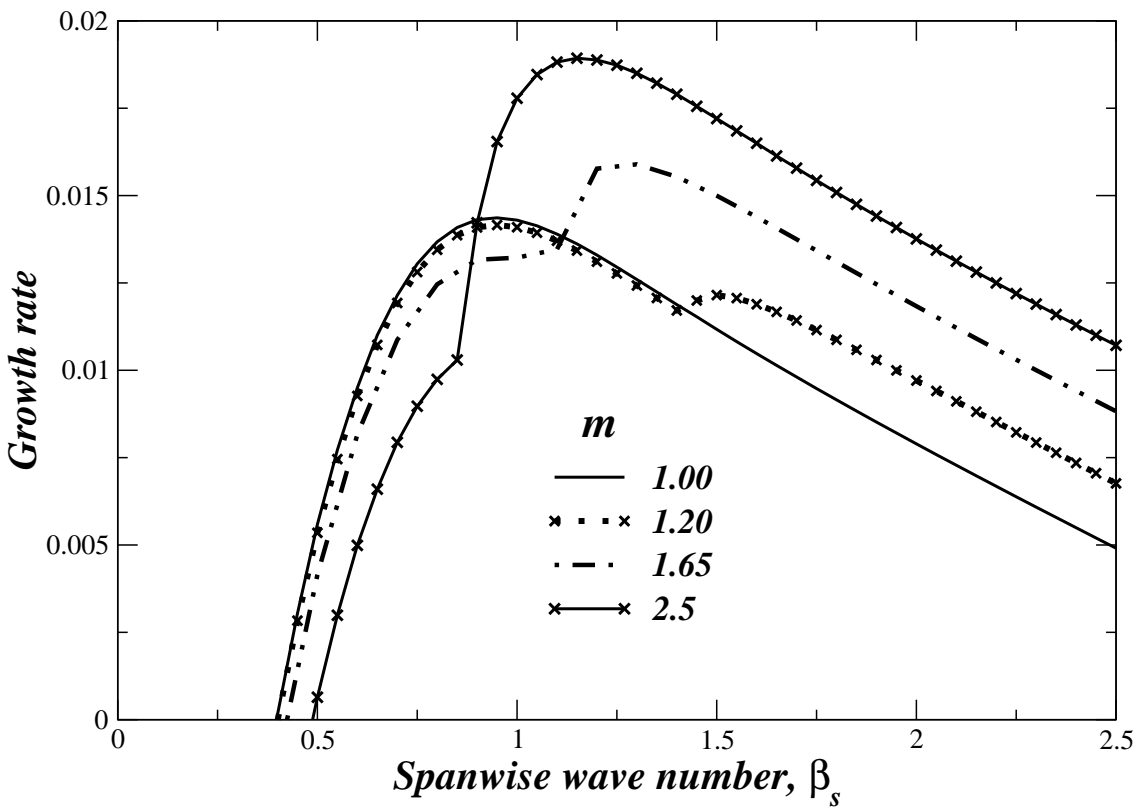

FIGURE 23. Asymmetric heating: dependence of growth rate on spanwise wave number of the secondary disturbance for various viscosity ratios, subharmonic case. $\alpha=1.0, A_{p}=0.01, R e=5000$.

to two-dimensional are now stabilised. A nonlinear or transient growth triggered by this new mode could mean that transition to turbulence proceeds somewhat differently, but further studies are needed to evaluate this.

While we have taken the amplitude of the primary mode to be constant, it is in fact, at these Reynolds numbers, a known slowly decaying function of time. Integrating instantaneous results over long times, we can compute the time dependence of amplitude of the secondary mode. This approach is a counterpart in time of the assumption of parallel flow in flows which vary slowly in $x$. The amplitude of the subharmonic secondary mode $A_{s}$ is shown as a function of time in figure 24 At low initial $A_{p}$, secondary modes are always stable while for higher $A_{p}$ significant growth is displayed up to large times. At low Reynolds number, the initial $A_{p}$ needed for a sustained secondary instability growth is very high. 


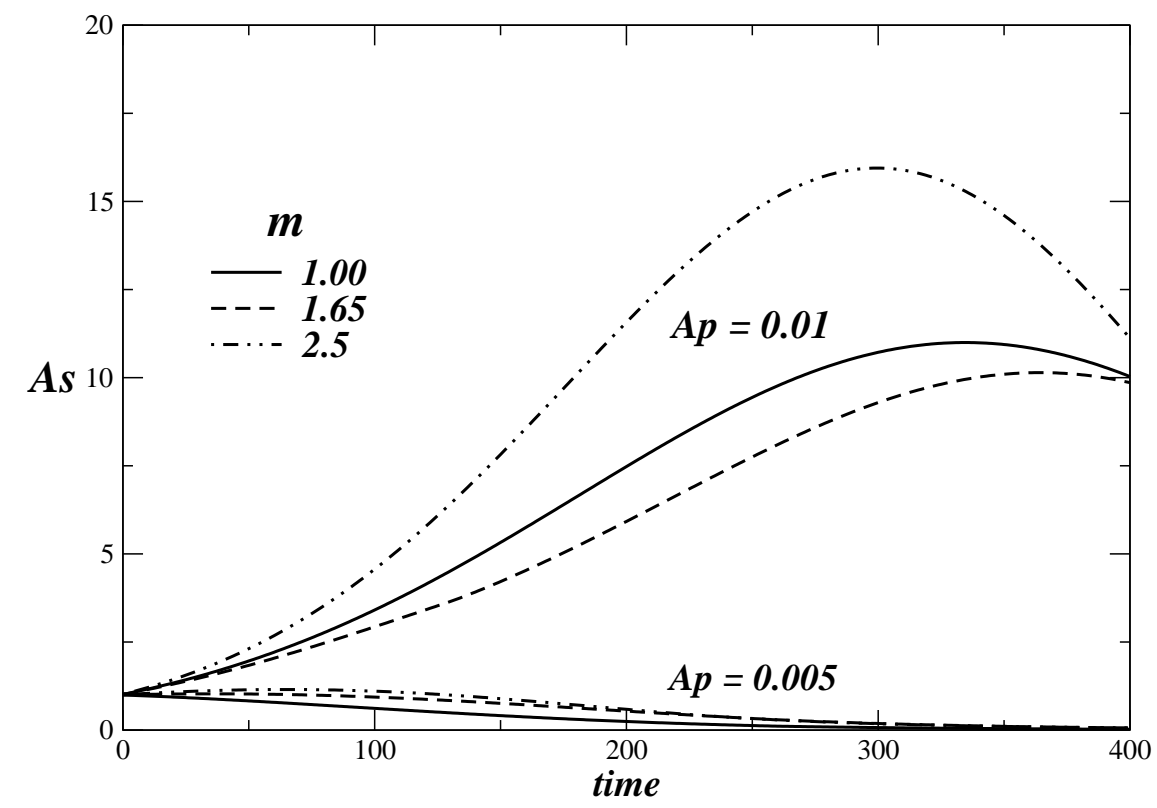

FIGURE 24. Asymmetric heating: variation with time of the amplitude of secondary disturbance for two sets of initial $A_{p} . \alpha=1.0, \beta_{s}=1.0, R e=5000$, Subharmonic mode.

In figure 25 the phase shift $P_{S}=\omega_{p} \frac{\alpha_{+}}{\alpha}-\omega_{s}$ is shown as a function of the spanwise wave number. The phase locking of the subharmonic wave (i.e., where $P_{S}$ is zero) is achieved at an earlier $\beta_{s}$ than for the unstratified case.

\subsection{Symmetrically heated channel}

Figure 26] shows the secondary perturbation growth rate variation with spanwise wave number $\beta_{s}$. A stabilisation with increase in viscosity ratio, especially when $m>1$, is evident. This behaviour is remarkable, being counter intuitive and opposite to the behaviour of the primary instability mode. The phase locking behaviour is similar to the asymmetrically heated case.

We have seen that in linear disturbance growth, the mean velocity profile (via the $U^{\prime \prime}$ term) has a dominant role. In the case of secondary growth as well, Orszag \& Patera (1983) have argued that inviscid effects are dominant, and act through vortex stretching 


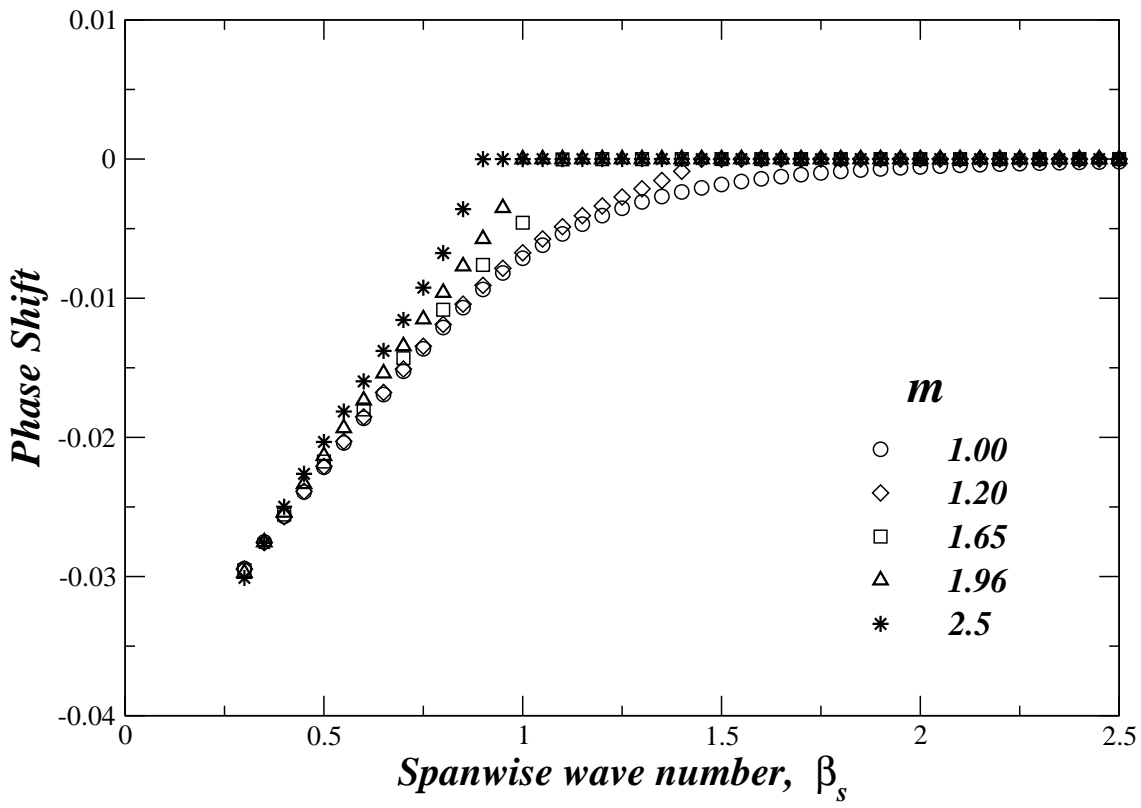

Figure 25. Asymmetric heating: variation of $P_{S}$ with spanwise wave number for various viscosity ratios, subharmonic case. $\alpha=1.0, A_{p}=0.01, R e=5000$.

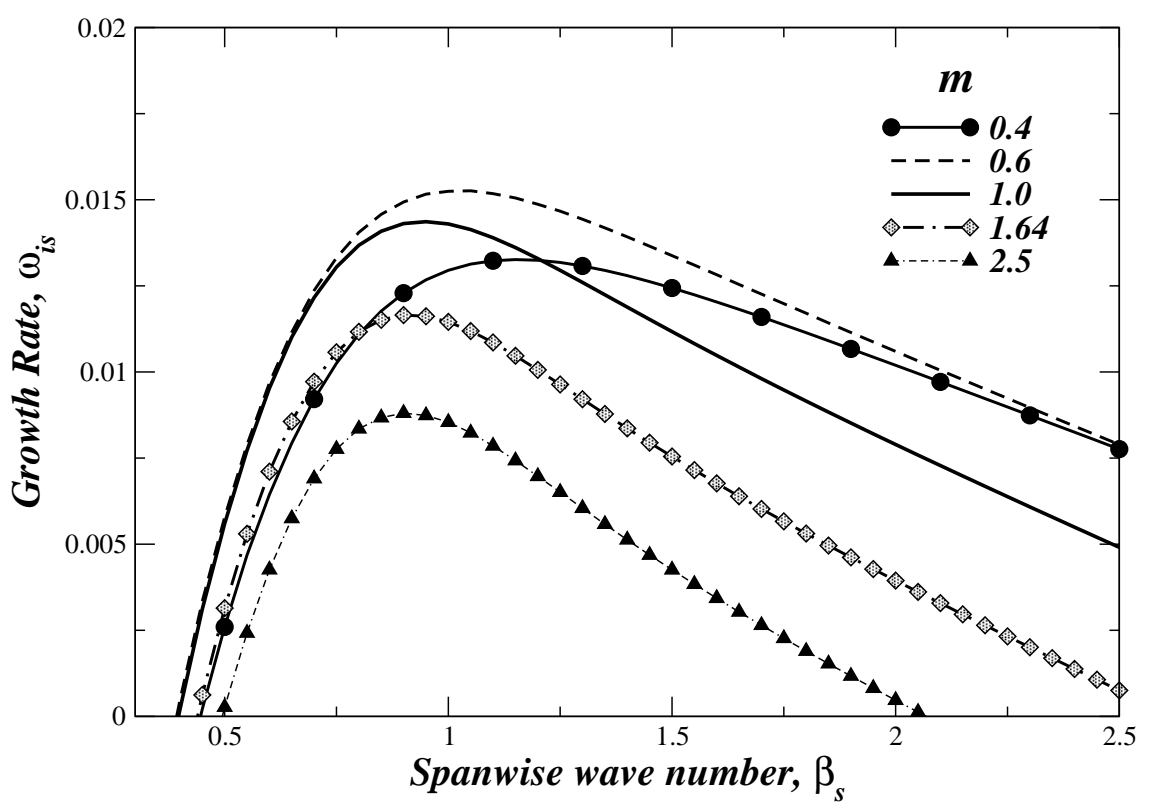

Figure 26. The dependence of growth rate on spanwise wave number of the secondary disturbance for various viscosity ratios, subharmonic case. $\alpha=1.0$, $A_{p}=0.01, R e=5000$, symmetric heating. 


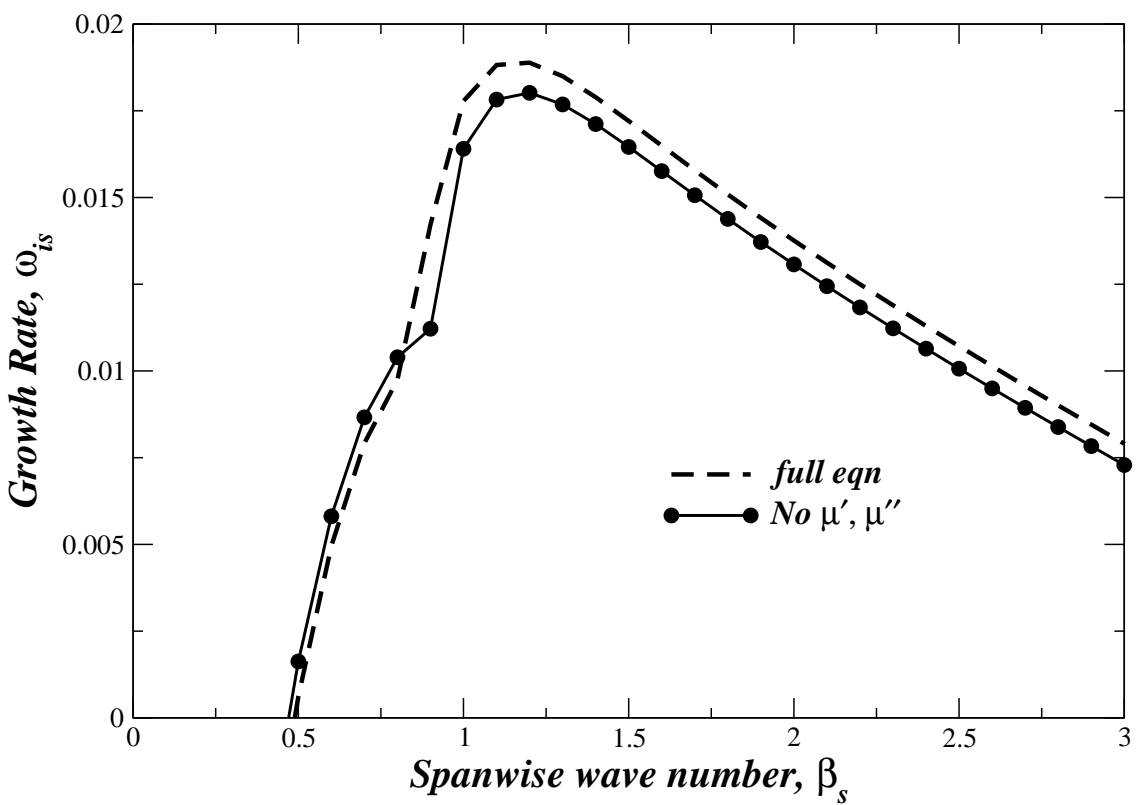

FiguRE 27 . The new mode of instability arises from changes in the velocity profile.

This is verified by switching off the viscosity gradient terms in the secondary stability equation. The dashed lines are for the full equation and the filled circles are with the viscosity gradient set to zero in the instability calculations, but retained in the mean flow computations. $\Delta T=50(m=2.5), \alpha=1.0, \alpha_{s}=0.5$, $A_{p}=0.01, R e=5000$, asymmetric heating.

and tilting. We are not able to make a conclusive statement on this, but it seems that heating affects secondary instability by an inviscid mechanism, through changes in the velocity profile. This is demonstrated in figure [27] where it is seen that switching off the viscosity gradient terms makes little difference to the result. Also, it is not evident why the sign of instability is opposite to that of primary modal growth. The relevant inference here is that viscosity stratification alone can have unexpected effects on the various mechanisms leading to transition to turbulence. 


\section{Conclusion}

The control of the flow using heating or cooling of the surface has long been practised, especially in open flows. In plane channel flow we have conducted a comprehensive study of the effect of heat, and show that there is no unique direction (either towards or away from stabilisation) in which the flow responds. Linear stability results are consistent with earlier studies in that the most unstable linear mode is suppressed when viscosity decreases towards the wall. Also the effect of Prandtl number is negligible. For Grashof number between about 3000 and 25000, separate modes of Rayleigh-Benard and Tollmien-Schlichting instability are evident, the former is at low Reynolds number. At higher levels of buoyancy, the modes merge.

The transient growth of disturbances is unaffected by viscosity stratification, but hugely increased by reduced heat diffusivity. Both of these are counter to the effect on the least stable linear mode. The Prandtl number is thus not an unimportant parameter, as was hitherto assumed. Transient growth is also very high in the presence of buoyancy of the appropriate sign. With increasing Prandtl and/or Grashof number, the growth is two-dimensional, not in streamwise streaks, which is quite unusual for transient growth.

Secondary instability of Tollmien-Schlichting waves is not considered an important player in the transition to turbulence in unheated channel flow, unless the free stream is unrealistically quiet. We show that a new destabilising mode appears with heating, for the case where viscosity is decreasing towards the wall!

It is hoped that this work with give impetus to experimental and computational studies to check these predictions and to explore wall heating in all its aspects as a control strategy for channel and pipe flows.

We are indebted to Prof. O.N. Ramesh for discussions throughout the course of this work and for reading the manuscript. A part of the work was carried out during the 
tenure of AS as a PhD student at the Indian Institute of Science. RG would like to thank

Defence R \& D Organisation, India for funding the project.

\section{REFERENCES}

Bayly, B. J., Orszag, S. A. \& Herbert, T. 1988 Instability mechanisms in shear-flow transition. Ann. Rev. Fluid Mech. 20, 359-391.

Biau, D. \& Bottaro, A. 2004 The effect of stable thermal stratification on shear flow instability. Phy. Fluids 16 (14), 4742-4745.

Chandrasekhar, S. 1961 Hydrodynamics and hydromagnetic stability. Oxford University Press, London.

Chikkadi, V. K., Sameen, A. \& Govindarajan, R. 2005 Preventing transition to turbulence: viscosity stratification will not always help. Phys. Rev. Lett. 95 (26), 264504.

Corbett, P. \& Bottaro, A. 2001 Optimal linear growth in swept boundary layers. J. Fluid Mech. 435, 1-23.

Craik, A. D. D. 1971 Non-linear resonant instability in boundary layers. J. Fluid Mech. 50, $393-413$.

Criminale, W. O., Jackson, T. L. \& Joslin, R. D. 2003 Theory and computation in hydrodynamic stability. Cambridge University Press.

Davies, S. J. \& White, C. M. 1928 An experimental study of the flow of water in pipes of rectangular section. Proc. Roy. Soc. (119), 92-107.

Deardorff, J. W. 1965 Gravitational instability between horizontal plates with shear. Phy. Fluid 8 (6), 1027-1030.

Faisst, H. \& Eckhardt, B. 2003 Traveling waves in pipe flow. Phys. Rev. Lett. 91, 224502.

Foster, R. 1997 Structure and energetics of optimal Ekman layer perturbations. J. Fluid Mech. 333, $97-123$.

Fujimura, K. \& Kelly, R. E. 1988 Stability of unstably stratified shear flow between parallel plates. Fluid Dyn. Res. 2, 281-292.

GAGE, K. S. 1971 The effect of stable thermal stratification on the stability of viscous parallel flow. J. Fluid Mech. 47 (1), 1-20. 
Gage, K. S. \& ReId, W. H. 1968 The stability of thermally stratified plane Poiseuille flow. J. Fluid Mech. 33 (1), 21-32.

Hanifi, A. \& Henningson, D. S. 1998 The compressible inviscid algebraic instability for streamwise independent disturbances. Phys. Fluids 10, 1784.

Herbert, T. 1983 Secondary instability of plane channel flow to subharmonic three-dimensional disturbances. Phys. Fluids 26 (4), 871-874.

Hof, B., van Doorne, C. W. H., Westerweel, J., Nieuwstadt, F. T. M., Faisst, H., Eckhardt, B., Wedin, H., Kerswell, R. R. \& WAleffe, F. 2004 Experimental observation of nonlinear traveling waves in turbulent pipe flow. Science 305, 1594-1598.

KAO, T. W. 1968 Role of viscosity stratification in the stability of two-layer flow down an incline. J. Fluid Mech. 33 (3), 561-572.

KAO, T. W. \& PARK, C. 1970 Experimental investigations of the stability of channel flows. part 1. flow of a single liquid in a rectangular channel. J. Fluid Mech. (43), 145.

KeRswelL, R. R. 2005 Recent progress in understanding the transition to turbulence in pipe. Nonlinearity 18, R17-R44.

Mahajan, R. L., Gebhart, B., Sammakia, B. \& Jaluria, Y. 1988 Buoyancy Induced Flows and Transport. Taylor and Francis.

Malik, V. S. \& Hooper, P. A. 2005 Linear stability and energy growth of viscosity stratified flows. Phys. Fluids 17, 024101.

Meseguer, A. 2002 Energy transient growth in the Taylor-Couette problem. Phys. Fluids $14(5)$.

Morkovin, M. V. \& Reshotko, K. 1989 Dialogue on progress and issues in stability and transition research. IUTAM symposium on Laminar-Turbulent transition pp. 3-29.

Narayanan, M. A. B. \& Narayanan, T. 1967 Z. Angew. Angew. Math. Phys. (18), 642.

Orszag, S. \& PAtera, A. 1983 Secondary instability of wall-bounded shear flows. J. Fluid Mech. 128, 347-385.

Orszag, S. A. 1971 Accurate solution of the Orr-Sommerfeld stability equation. J. Fluid Mech. 50, 689-703. 
Patel, V. C. \& Head, M. R. 1969 Some observations on skin friction and velocity profiles in fully developed pipe and channel flows. J. Fluid Mech. (38), 181.

Pinarbasi, A. \& Liakopoulos, A. 1995 Role of variable viscosity in the stability of channel flow. Int. Comm. Heat Mass Transfer 22(6), 837-847.

Platten, J. K. \& Legros, J. C. 1984 Convection in liquids. Springer-Verlag.

Potter, M. C. \& Graber, E. 1972 Stability of plane Poiseuille flow with heat transfer. Phys. Fluids 15, 387 .

Reddy, S. C. \& Henningson, D. S. 1993 Energy growth in viscous channel flows. J. Fluid Mech. 252, 209-238.

Reddy, S. C. \& Henningson, D. S. 1994 On the role of linear mechanisms in transition to turbulence. Phys. Fluids 6, 1396.

Reddy, S. C., Schmid, P. J., Bagget, J. S. \& Henningson, D. S. 1998 On stability of streamwise streaks and transition thresholds in plane channel flows. J. Fluid Mech. 365, 269-303.

Sameen, A. 2004 Stability of plane channel flow with viscosity-stratification. PhD thesis, Dept. of Aerospace Engg., Indian Institute of Science.

Schafer, P. \& Herwig, H. 1993 Stability of plane Poiseuille flow with temperature dependent viscosity. Int. J. Heat Mass Transfer 36 (9), 2441-2448.

Schafer, P., Severin, J. \& Herwig, H. 1995 The effect of heat transfer on the stability of laminar boundary layers. Int. J. Heat Mass Transfer 38 (10), 1855.

Schmid, P. J. \& Henningson, D. S. 2001 Stability and Transition in Shear Flows. SpringerVerlag, New York.

Strazisar, A. J., Reshotko, E. \& Prahl, J. M. 1977 Experimental study of the stability of heated laminar boundary layer in water. J. Fluid Mech. 83 (2), 225-247.

Turner, J. S. 1959 Buoyant plumes and thermals. Ann. Rev. Fluid Mech. 1, 29-44.

Tveitereid, M. 1974 On the stability of thermally stratified plane Poiseuille flow. ZAMM 54, 533-540.

WALEFfe, F. 1995 Hydrodynamic stability and turbulence: Beyond transients to a selfsustaining process. SIAM 95, 319-343. 
Wall, D. P. \& Wilson, S. K. 1996 The linear stability of channel flow of fluid with temperature-dependent viscosity. J. Fluid Mech. 323, 107-132.

Wazzan, A. R., Gazley, C. \& Smith, A. M. O. 1979 Tollmien-Schlichting waves and transition. Prog. Aerospace Sci. 18 (2), 351-392.

Wazzan, A. R., Okamura, T. T. \& Smith, A. M. O. 1968 The stability of water flow over heated and cooled flat plates. J. Heat Transfer 99, 109-114.

Zhang, J., Childress, S. \& Libchaber, A. 1998 Non-Boussinesq effect: asymmetric velocity profiles in thermal convection. Phys. Fluids 10 (6), 1534-1536. 